\title{
ON SOLUTION OF PARTIAL DIFFERENTIAL EQUATIONS BY THE HAHN-BANACH THEOREM
}

\author{
BY \\ P. R. GARABEDIAN AND MAX SHIFFMAN
}

1. Introduction. In this paper we show how to construct the Green's and Neumann's functions of a linear elliptic partial differential equation by a method based on linear functionals. The construction depends essentially on the Hahn-Banach extension theorem [1], which has been used recently in a similar connection by Lax [5]. Lax's approach differs from ours in that, while his proof centers about a bounded linear functional based on inhomogeneous boundary conditions, our proof centers rather about a functional based on the solution of an inhomogeneous differential equation. This latter point of view has the advantage that in constructing the Green's and Neumann's functions we need only a fundamental solution of the differential equation in the small, and, indeed, we can succeed with merely a parametrix, which is readily obtained for a linear elliptic partial differential equation, whereas all earlier existence proofs of the type studied here $[3 ; 4 ; 5]$ are based on the previous knowledge of a fundamental solution in the large. Our considerations are also advantageous for domains with general boundaries and for Riemannian manifolds.

For earlier work on application of the Hahn-Banach theorem to boundary value problems, we refer to the papers of Caccioppoli [2] and of Miranda [6]. It should be mentioned at the outset that the proof of the Hahn-Banach theorem in the form in which we shall need it in this paper does not require transfinite induction, since the Banach space of continuous functions which we use is separable.

2. The Neumann's function. Let $P=P(z) \geqq \epsilon>0$ be a positive, continuously differentiable function of the point $z$ in a plane domain $D$ bounded by a finite number of simple closed analytic curves $C$. Denote by $B$ the Banach space of all functions $f$ which are continuous in $D+C$, with the norm

$$
\|f\|=\max |f| \text {, }
$$

and denote by $B^{\prime}$ the subspace of $B$ of functions

$$
f=\Delta u-P u,
$$

where $u$ is any function, continuous with its first and second derivatives in $D+C$, whose inner normal derivative vanishes on $C$,

$$
\frac{\partial u}{\partial \nu}=0
$$

Presented to the Society, November 28, 1953; received by the editors January 19, 1953. 
For any point $w$ in $D$, we define a linear functional $L_{w}\{f\}$ on $B^{\prime}$ by the formula

$$
u(w)=L_{w}\{\Delta u-P u\} .
$$

That this linear functional is uniquely defined and bounded can be shown by the following argument.

First, if

$$
\Delta U-P U \leqq 0
$$

in $D$ and

$$
\frac{\partial U}{\partial \nu}=0
$$

on $C$, then $U \geqq 0$ in $D$. For if $U<0$ in some subregion of $D$, we could apply Green's theorem to that subregion to obtain

$$
\iint\left[(\nabla U)^{2}+P U^{2}\right] d \tau=-\iint U[\Delta U-P U] d \tau-\int U \frac{\partial U}{\partial \nu} d s
$$

where $d \tau$ is the area element and where $s$ denotes the arc length along the boundary curves of the region $U<0$. By (4), (5), and the condition $U<0$, it is clear that the right-hand side of (6) is nonpositive, whereas it is equally clear that the left-hand side of (6) is positive. This contradiction yields the original statement $U \geqq 0$. Uniqueness of the functional $L_{w}$ for given $\Delta u-P u$, as defined in (3), is an immediate consequence of this.

Second, let $v_{1}=1 / \epsilon$, so that

$$
\Delta v_{1}-P v_{1} \leqq-1
$$

and let $M=\|f\|$. Then in $D$

$$
\Delta\left(M v_{1}-u\right)-P\left(M v_{1}-u\right) \leqq-M-f=-\|f\|-f \leqq 0,
$$

and on $C$

$$
\frac{\partial}{\partial \nu}\left(M v_{1}-u\right)=0
$$

whence $M v_{1}-u \geqq 0$. It follows from the definition (3) that for $f$ in $B^{\prime}$,

$$
L_{w}\{f\} \leqq \frac{1}{\epsilon}\|f\|,
$$

which proves that $L_{w}$ is a bounded functional.

We conclude from the Hahn-Banach theorem that the bounded linear functional $L_{w}$ can be extended from the vector space $B^{\prime}$ to operate on all elements of the Banach space $B$ and to satisfy (8) there. By applying the ex- 
tended functional in a suitable way, we shall set up an expression which we shall prove to be the Neumann's function of the linear elliptic partial differential equation

$$
\Delta u-P u=0
$$

in $D$.

For the construction, we need a so-called parametrix $S(w, z)$ for (9). Such a paramatrix is a function of $w$ which has a logarithmic singularity at $w=z$ and is such that $\Delta S-P S$ is continuous, even at the point $z$. More precisely, we shall require certain regularity properties of $\Delta S-P S$ as a function of $z$, and for our present purposes we select $S(w, z)$ to be a symmetric function of $w$ and $z$. Let

$$
g(w, z)=\log \frac{1}{|w-z|}+\cdots
$$

be a fundamental solution of Laplace's equation in a region $E$. When $E$ is a semicircle, it is an elementary problem to construct $g$ explicitly in such a way that $g$ vanishes on the circular arc bounding $E$ and has zero normal derivatives on the diameter bounding $E$. We set

$$
\begin{aligned}
& I_{1}(w, z)=\frac{1}{2 \pi} \iint_{E} P(t) g(w, t) g(z, t) d \tau, \\
& I_{2}(w, z)=\frac{1}{2 \pi} \iint_{E} P(t) g(w, t) I_{1}(z, t) d \tau,
\end{aligned}
$$

and we set

$$
S_{0}(w, z)=g(w, z)-I_{1}(w, z)+I_{2}(w, z),
$$

whence by Poisson's equation

$$
\Delta S_{0}-P S_{0}=-P I_{2},
$$

which shows, for our purposes, that $S_{0}$ is a parametrix for (9). If $E$ is the above semicircle and $g$ is explicitly constructed in the manner described for a semicircle, then $S_{0}$ will have a normal derivative which vanishes on the diameter bounding $E$. For $z$ in the neighborhood of a point $z_{0}$ in which we are interested, we obtain for all $w$ a suitable parametrix $S$ whose normal derivative vanishes on $C$ by multiplying $S_{0}$ by a function $\delta$, with continuous derivatives of the first few orders, which is identically 1 near $z_{0}$, but which vanishes identically outside a small circle about $z_{0}$. For example, with sufficiently small $\epsilon>0$, we can take $\delta=f_{\epsilon}\left(\left|z-z_{0}\right|\right) f_{\epsilon}\left(\left|w-z_{0}\right|\right)$, where $f_{\epsilon}(r)$ is a continuous function of $r$ with continuous derivatives of the first few orders which is equal to 1 for $0 \leqq r \leqq \epsilon$ and vanishes for $r \geqq 2 \epsilon$.

Having obtained a parametrix $S(w, z)$ in this manner, we define the Neu- 
mann's function $N(w, z)$ for (9) in $D$ by the formula

$$
N(w, z)=S(w, z)-L_{w}\{[\Delta-P(t)] S(t, z)\},
$$

and we proceed to show that $N$ has, as a function of the point $z$, the characteristic properties of the Neumann's function. It is easily verified from the definition (3) that $N$ is independent of the choice of the parametrix $S$, since the normal derivative of $S$ vanishes on $C$ and the difference of two such parametrices is regular.

First, since $L_{w}$ is a bounded functional, the quantity $L_{w}[\{\Delta-P(t)\} S(t, z)]$ depends continuously on $z$, and, indeed, we can differentiate with respect to $z$ under the functional $L_{w}$. We obtain

$$
\begin{aligned}
{[\Delta-P(z)] N(w, z)=} & {[\Delta-P(z)] S(w, z) } \\
& -L_{w}\{[\Delta-P(t)][\Delta-P(z)] S(t, z)\}=0 .
\end{aligned}
$$

The expression on the right vanishes, according to (3), because, as a function of $w$, the expression $[\Delta-P(z)] S(w, z)$ has continuous second derivatives in $D+C$ and has a normal derivative which vanishes on $C$. Thus $N$ satisfies the partial differential equation (9) as a function of $z$.

Second, $N(w, z)$ has a logarithmic singularity at the point $z=w$, since the second term on the right in (12) is bounded.

Third, we can calculate the normal derivative of $N$ at a point $z_{0}$ of $C$ conveniently by making a special choice of $E$ and $g$ in (11). The curves $C$ have an analytic representation $z=z(s)$ in terms of a parameter $s$, and we choose $E$ to lie in $D$ and to correspond in the $s$-plane to a small semicircle whose bounding diameter lies on the real axis and whose center maps into $z_{0}$. We choose $g$ to be the fundamental solution of the Laplace equation in the semicircle which vanishes on the circular boundary and has normal derivative zero on the rectilinear boundary. Since $z=z(s)$ is a conformal mapping, $g$ satisfies Laplace's equation in $E$ also, and substitution into (11) yields a parametrix $S_{0}$ with vanishing normal derivatives with respect to both $z$ and $w$ on an arc of $C$ including the point $z_{0}$. Multiplication by a function $\delta$ which is identically 1 in a small neighborhood of $z_{0}$, identically zero outside a slightly larger neighborhood of $z_{0}$, and has a vanishing normal derivative on $C$, yields our choice of $S$. (A selection of $\delta$ as a function of $|s|$ will satisfy these conditions.)

With this choice of $S$ in (12), it is permissible to compute the normal derivative of $N$ with respect to $z$ at $z_{0}$ by differentiating under the bounded functional $L_{w}$. We find

$$
\frac{\partial N}{\partial \nu}=\frac{\partial S}{\partial \nu}-L_{w}\left\{(\Delta-P) \frac{\partial S}{\partial \nu}\right\}=0,
$$

since $\partial S / \partial \nu=\delta \cdot \partial S_{0} / \partial \nu \equiv 0$ throughout $D$.

We have thus shown that $N$ has the three characteristic properties of the 
Neumann's function of (9) in $D$, as a function of $z$, and we conclude therefore that the Neumann's function exists and is equal to $N$.

We remark that it is possible to generalize the above existence proof to partial differential equations other than (9). Also, the restriction to two independent variables is unnecessary, and, indeed, for more independent variables the only additional complication comes in deriving the boundary condition (14), which has been based here on conformal mapping. However, for general elliptic equations in several variables we can treat the easy case of a plane boundary without any loss of generality, since we can always find a transformation of coordinates which takes a small portion of an analytic surface into a portion of a plane and this transformation will merely carry one elliptic equation into another which can be discussed equally well. In another direction, we can generalize the existence proof by replacing the boundary condition (2) by

$$
\frac{\partial u}{\partial \nu}-\lambda u=0
$$

where $\lambda$ is a given positive function on $C$. In this case, we obtain the existence of a Green's function of the third kind. Also, we note that the domain $D$ may be a multisheeted Riemann surface over the plane, instead of a schlicht region.

3. The Green's function. In this section we study the Dirichlet problem for a linear elliptic partial differential equation in several independent variables by a technique analogous to that of $\S 2$. Our objective is to give a more penetrating analysis of the boundary condition, with hypotheses on the boundary far less restrictive than analyticity. This can be achieved by appropriate use of the principle of majorization and by a stronger use of the HahnBanach theorem. We present the material for three independent variables in space for the sake of simplicity, and we merely remark that for any finite number of dimensions the steps to be carried out are similar.

Let $D$ be a bounded domain in space with boundary $C$ having the following property: Each point of $C$ can be touched by a solid sphere $R$ which is otherwise disjoint from $D+C$. Let $P(z) \geqq 0$ be a non-negative continuously differentiable $\left.{ }^{1}\right)$ function of the point $z$ in a neighborhood of $D+C$, and let $B^{\prime}$ denote the linear space of all functions

$$
f=\Delta u-P u
$$

in $D+C$ with $u$ twice continuously differentiable in $D+C$ and zero on $C$. We let $B$ denote the Banach space of all continuous functions in $D+C$ with the norm

$$
\|f\|=\max |f| \text {. }
$$

(1) The considerations apply if, in place of continuous derivatives, $P(z)$ satisfies a Lipschitz condition $\left|P(z)-P\left(z^{\prime}\right)\right| \leqq K\left|z-z^{\prime}\right|$. 
For any point $w$ in $D$ we define a linear functional $L_{w}$ over $B^{\prime}$ by the formula

$$
L_{w}[\Delta u-P u]=u(w) .
$$

That this functional is uniquely defined follows from the theorem that a solution of $\Delta u-P u=0$ in $D$ which vanishes on $C$ must be identically 0 , since it cannot have a positive maximum or negative minimum in the interior of $D$.

We prove that, for $f$ in $B^{\prime}$,

$$
L_{w}\{f\} \leqq b(f),
$$

where

$$
b(f)=\inf v(w)
$$

among all twice continuously differentiable functions $v$ with

$$
\Delta v-P v \leqq f
$$

in $D$, and $v \geqq 0$ on $C$. For the proof, we note that since

$$
\Delta(v-u)-P(v-u) \leqq f-f=0,
$$

$v-u$ cannot have a negative minimum inside $D$. Therefore, since $v-u \geqq 0$ on $C$, we must have $v-u \geqq 0$ in $D$ also, which yields the desired estimate (17). In fact, since $u$ is itself an admissible function in the determination of $b[f]$, we have equality in (17) for $f$ in $B^{\prime}$. In particular, $b[0]=0$.

The functional $b[f]$ defined in (18), (19) has a meaning for any $f$ in the entire space $B$. For, let $v_{2}$ be the function

$$
v_{2}=K-\frac{1}{6}\left(x^{2}+y^{2}+z^{2}\right),
$$

where $K$ is a large constant such that $v_{2} \geqq 0$ in $D+C$. We have

$$
\Delta v_{2}-P v_{2} \leqq-1 \text {, }
$$

and $\|f\| v_{2}$ is an admissible function for (18). Thus

$$
b[f] \leqq v_{2}(w) \cdot\|f\| \leqq K \cdot\|f\| .
$$

Also, we notice that

$$
b[f] \leqq 0
$$

if $f \geqq 0$ throughout $D$, since the function $v \equiv 0$ is then admissible in (18).

The functional $b[f]$ is positively homogeneous and subadditive; i.e.,

$$
b[t f]=t b[f] \text { if } t>0, \quad b\left[f_{1}+f_{2}\right] \leqq b\left[f_{1}\right]+b\left[f_{2}\right] .
$$

The latter inequality follows from the fact that if $v^{\prime}$ and $v^{\prime \prime}$ are admissible functions for the determination of $b\left[f_{1}\right]$ and $b\left[f_{2}\right]$, respectively, then $v^{\prime}+v^{\prime \prime}$ is admissible for $b\left[f_{1}+f_{2}\right]$. We remark that since we already know that $b[0]$ $=0$, the subadditivity shows that $b[f] \geqq-b[-f] \geqq-K\|f\|$, whence $b[f]$ is 
finite and likewise bounded from below.

We now use the Hahn-Banach theorem to extend $L_{w}[f]$ as a linear functional over the entire space $B$ still satisfying the inequality (17). Applying the extended functional to a parametrix for the equation

$$
\Delta u-P u=0
$$

in a suitable way, we shall obtain the Green's function in $D$ for this equation.

Let $E$ be a (small) region containing $z$, and let $g(w, z)=1 /|w-z|+\cdots$ be a fundamental solution for Laplace's equation which is non-negative in $E$ and symmetric in $w$ and $z(|w-z|$ means the distance between $w, z)$. For example, $g(w, z)$ could be the Green's function for Laplace's equation for a sphere enclosing $E$. Set

$$
I_{j}(w, z)=\frac{1}{4 \pi} \iiint_{E} P(t) g(w, t) I_{j-1}(z, t) d \tau,
$$

$j=1,2, \cdots$, where $I_{0}(w, z)=g(w, z)$ and $d \tau$ is the volume element. We have $I_{j}(w, z) \geqq 0$ in $E$. Then

$$
S_{0}(w, z)=\sum_{j=0}^{3}(-1)^{j} I_{j}(w, z)
$$

is a parametrix for the equation $\Delta u-P u=0$, and

$$
\Delta S_{0}-P S_{0}=P I_{3} \geqq 0 .
$$

For $z$ in the neighborhood of a point $z_{0}$ in which we are interested, we obtain for all $w$ in $D$ a suitable parametrix $S_{1}(w, z)$ by multiplying $S_{0}$ by a function $\delta$, with continuous derivatives of the first few orders, which is identically 1 near $z_{0}$, but which vanishes identically outside a small sphere about $z_{0}$.

We now define the Green's function $G(w, z)$ in $D$ by the formula

$$
\left.G(w, z)=S(w, z)-L_{w} \mathrm{~L}\{\Delta-P(t)\} S(t, z)\right],
$$

where $S(w, z)$ is a parametrix in $D$ which vanishes when $w$ is on $C$. We show directly that $G$ has as a function of $z$ in $D$ the characteristic properties of the Green's function. Note that the formula (25) is independent of the particular $S(w, z)$ used, by virtue of (16).

First, the second term on the right in (25) is bounded for $z$ near $w$ by virtue of (17) and (20), so that $G$ must have a pole at $z=w$. Second, since the functional $L_{w}$ is a bounded linear functional, the second term on the right in (25) is continuous in $z$ for $z$ in $D$. Indeed, we can differentiate with respect to the coordinates of $z$ under the operator $L_{w}$, and we find

$$
\{\Delta-P(z)\} G=\{\Delta-P(z)\} S-L_{w}[\{\Delta-P(t)\}\{\Delta-P(z)\} S]=0 .
$$

The last equality follows from (16), since $\{\Delta-P(z)\} S(w, z)$ is a twice con- 
tinuously differentiable function of $w$ which vanishes for $w$ on $C$.

Third, we prove that $G \geqq 0$. Let $\sigma$ be a large positive number and let $F(S)$ be a function of $S$ which is identically $S$ for $S \leqq \sigma$, which is continuous with its first two derivatives for all $S$, which has a finite limit as $S \rightarrow+\infty$, and which has a nonpositive second derivative $F^{\prime \prime}(S)$ for $S>\sigma$. With the parametrix $S_{0}$ constructed in (23), we have $\left({ }^{2}\right)$

$$
\begin{aligned}
\{\Delta-P\} F\left(S_{0}\right)-\{\Delta-P\} S_{0}= & F^{\prime \prime}\left(\nabla S_{0}\right)^{2}+\left(F^{\prime}-1\right)\left(\Delta S_{0}-P S_{0}\right) \\
& +\left(F^{\prime} S_{0}-F\right) P \leqq 0
\end{aligned}
$$

by (24) and the inequalities $F^{\prime} S-F \leqq 0$ and $F^{\prime}-1 \leqq 0$, which follow from $F^{\prime \prime} \leqq 0$.

Using the parametrix $S_{1}$ constructed in the paragraph immediately following (24), we therefore have for sufficiently large $\sigma$

$$
(\Delta-P) F\left(S_{1}\right)-(\Delta-P) S_{1} \leqq 0 .
$$

It now follows that

$$
F\left(S_{1}\right)=L_{w}\left[(\Delta-P) F\left(S_{1}\right)\right] \geqq L_{w}\left[(\Delta-P) S_{1}\right],
$$

since the linear functional $L_{w}[f] \leqq 0$ when $f$ is everywhere non-negative, by virtue of (21) and (17). Therefore

$$
G=S_{1}-L_{w}\left[(\Delta-P) S_{1}\right] \geqq S_{1}-F\left(S_{1}\right) \geqq 0,
$$

which is the desired result.

Finally, we prove that $G$ has boundary values 0 . Let $z_{0}$ be a point of $C$, let $R$ be a small sphere in the exterior of $D$ and touching $C$ at $z_{0}$, and let $R^{\prime}$ be a small sphere containing $z_{0}$ and orthogonal to $R$. Denote the region outside $R$ and inside $R^{\prime}$ by $E$, and let $g(w, z)$ be the Green's function for Laplace's equation in $E$. For this choice of $E$ and $g$, define $I_{j}(w, z)$ as in (22) and set

$$
S_{0}^{*}(w, z)=\sum_{j=0}^{4}(-1)^{i} I_{j}(w, z) .
$$

Then,

$$
\Delta S_{0}^{*}-P S_{0}^{*}=-P I_{4}(w, z) \leqq 0 .
$$

Also, for $w$ on the boundary of $E$, we have $g(w, z)=0$ and $I_{j}(w, z)=0$ from (22). Therefore, $S_{0}^{*}(w, z)=0$ on the boundary of $E$. Since $S_{0}^{*}$ cannot have a negative minimum in the interior of $E$ by (27), it follows that

$$
S_{0}^{*} \geqq 0
$$

throughout $E$.

(2) For the general differential equation, $F$ can always be constructed so that the first term corresponding to $F^{\prime \prime}\left(\nabla S_{0}\right)^{2}$ is the dominant term, and we still have the inequality. 
Multiply $S_{0}^{*}$ by a non-negative function $\delta$, continuous with its first few derivatives, which is identically 1 in a small neighborhood of $z_{0}$ and which is identically 0 in a slightly larger neighborhood of $z_{0}$. This yields a parametrix $S_{1}^{*}$ throughout $D$ which is non-negative on $C$ and approaches 0 as $z \rightarrow z_{0}$.

For any parametrix $S$ which vanishes on $C$, the difference $S_{1}^{*}-S$ is twice continuously differentiable in $D$ and is non-negative on $C$. Hence, by (17), (18), and (19), with $f=(\Delta-P)\left(S_{1}^{*}-S\right)$ and $v=S_{1}^{*}-S$, we obtain

$$
L_{w}\left[(\Delta-P)\left(S_{1}^{*}-S\right)\right] \leqq S_{1}^{*}-S,
$$

or

$$
G \leqq S_{1}^{*}-L_{w}\left[(\Delta-P) S_{1}^{*}\right] .
$$

Letting $z \rightarrow z_{0}$, we have $S_{1}^{*}(w, z) \rightarrow 0$ and $(\Delta-P(t)) S_{1}^{*}(t, z) \rightarrow 0$ uniformly in $t$; this last limiting relation is clear outside any neighborhood of $z_{0}$, while inside a small neighborhood of $z_{0}$ it follows from (27). Consequently

$$
\limsup _{z \rightarrow z_{0}} G(w, z) \leqq 0,
$$

and since $G \geqq 0$, we can make the final desired conclusion

$$
\lim _{z \rightarrow C} G(w, z)=0 .
$$

These calculations show that $G$ is the Green's function of $D$ for the linear elliptic partial differential equation $\Delta u=P u$. This construction of $G$ gives a remarkably simple proof of the existence of the Green's function, without previous knowledge of a fundamental solution in the large. In the special case $P \equiv 0$ we obtain the Green's function of Laplace's equation.

Incidentally, the symmetry of the Green's function is also contained in the above. Since $G(w, z)$ is the Green's function for $\Delta u=P u$ as a function of the second variable, with singularity at the first variable, the function $G(z, w)$ is an admissible singularity function $S(w, z)$ in (25). We immediately obtain, therefore,

$$
G(w, z)=G(z, w) .
$$

Of course, solvability of the Dirichlet problem is obtained once $G$ is known.

4. General domains. The argument of the preceding section can easily be generalized to the case of a domain $D$, bounded or unbounded, with an arbitrary boundary $C$. Let $D$ lie in $n$-dimensional Euclidean space, where $n \geqq 3$, and let $P(z) \geqq 0$ be an arbitrary non-negative function over $D$, continuous with continuous first derivatives in $D$. In 2 dimensions, we suppose either that $D$ is bounded, or if $D$ is unbounded, that $P(z)>0$. Consider the function

$$
v_{3}=g(|z|),
$$


where $|z|=r$ is the distance from $z$ to the origin, and

$$
g(r)=\int_{r}^{\infty} \frac{r d r}{1+r^{n}}
$$

so that

$$
\Delta g(r)=-\frac{n}{\left(1+r^{n}\right)^{2}}<0 .
$$

The function $v_{3}$ is positive, is continuous and has continuous first and second derivatives, and

$$
-w_{3}=(\Delta-P) v_{3}<0,
$$

so that $w_{3}>0$. In 2 dimensions, with $D$ unbounded and $P>0$, select $v_{3} \equiv 1$, and $w_{3}=P>0$. Define the norm of a function $f$ which is continuous in $D$ by

$$
\|f\|=\sup \left|\frac{f}{w_{3}}\right| \text {, }
$$

and let $B$ denote the Banach space of the functions $f$ with finite norm. Let $B^{\prime}$ denote the subspace of functions $f$ in $B$ given by

$$
f=\Delta u-P u,
$$

where $u$ is continuous together with first and second derivatives in $D$, and $u \rightarrow 0$ as the boundary or the point at infinity (if $D$ is unbounded) is approached. Finally, define the linear functional $L_{w}$ over $B$ as in (16).

Proceeding as in $\S 3$, define the bound $b[f]$ for any $f$ in $B$ as in (18), (19), with the condition $v \geqq 0$ on $C$ replaced by $\lim \inf v \geqq 0$ as the boundary $C$ or the point at infinity is approached. Inequality (17) applies. An admissible function in (19) is $\|f\| v_{3}$. For,

$$
(\Delta-P)\|f\| v_{3}=-\|f\| w_{3} \leqq-\frac{|f|}{w_{3}} w_{3} \leqq f .
$$

Thus

$$
b[f] \leqq v_{3}(w)\|f\|
$$

as in (20), and we also have (21).

Now extend the functional $L_{w}[f]$ throughout $B$ so that the inequality (17) is retained, and define the function $G(w, z)$ as in $\$ 3$ by $(25)$, where $S(w, z)$ is a parametrix which vanishes on $C$ and at infinity. $\$ 3$ shows that $G(w, z)$, as a function of $z$, satisfies the equation

$$
\Delta u-P u=0,
$$

and has a fundamental singularity at $z=w$. As a function of the second vari- 
able $z, G$ is therefore a fundamental solution over $D$ of $\Delta u=P u$.

We shall prove that, among all fundamental solutions, $G$ is characterized by the property of being the smallest positive fundamental solution. This replaces the condition $G=0$ on the boundary $C$ because of the arbitrariness of $C$.

First, $G(w, z) \geqq 0$ throughout $D$, just as in $\S 3$. Second, we shall show that the important symmetry property

$$
G(w, z)=G(z, w)
$$

is easily contained in our formulation. Let $S^{\prime}(w, z)$ be a parametrix with nonnegative boundary values and non-negative values at infinity (i.e., let $\lim \inf S^{\prime}(w, z) \geqq 0$ as $w \rightarrow C$ or the point at infinity, if $D$ is unbounded). Then, by (17) and (18),

$$
S^{\prime}(w, z)-S(w, z) \geqq L_{w}\left[(\Delta-P)\left(S^{\prime}(t, z)-S(t, z)\right)\right],
$$

or

$$
S^{\prime}(w, z)-L_{w}\left[(\Delta-P) S^{\prime}(t, z)\right] \geqq G(w, z) .
$$

Now, we have already proved that $G$, as a function of its second point, is a fundamental solution with singularity at the first point. Thus, $G(z, w)$ (note the location of $z$ and $w$ ) is a function of $w$ which is continuous and has continuous first and second derivatives throughout $D$, except at the point $z$. Since $G(z, w) \geqq 0$, the function $G(z, w)$ is therefore admissible as a choice for the parametrix $S^{\prime}(w, z)$ in (34), and we obtain

$$
G(z, w) \geqq G(w, z) .
$$

This inequality holds for any location of the points $z, w$, so that by interchanging $z, w$ the reverse inequality follows and the symmetry property (33) is established.

The symmetry property (33) shows that $G(w, z)$ is a well-behaved function of its first point $w$ as well, and is a fundamental solution as a function of this first point with singularity at the second point.

Third, $G(w, z)$ is the smallest positive fundamental solution in $D$ with singularity at $z$. For, if $F(w, z)$ is such a positive fundamental solution, it can be used as the parametrix $S^{\prime}(w, z)$ in (34), with the desired result

$$
F(w, z) \geqq G(w, z) .
$$

We mention the following additional property of $G(w, z)$ : If we exclude a neighborhood $\Omega$ of the singular point $z$, the function $G(w, z)$ is bounded for $w$ outside this neighborhood. If this were not so, use the function $F\left(S_{0}\right)$ constructed in connection with (26), for sufficiently large $\sigma$. Define the function $\phi(w, z)$ as equal to $G(w, z)$ for $w$ in $\Omega$, and equal to $F(G(w, z))$ for $w$ outside $\Omega$. The function $\phi(w, z)$ is an admissible parametrix in (34) with the result 


$$
\phi(w, z)-L_{w}[(\Delta-P) \phi(t, z)] \geqq G(w, z) .
$$

But $(\Delta-P) \phi(t, z) \leqq 0$ by (26), and (21) now gives

$$
\phi(w, z) \geqq G(w, z) .
$$

Selecting a point $w$ outside $\Omega$ where $\phi(w, z) \neq G(w, z)$, we contradict the inequality $F\left(S_{0}\right)<S_{0}$, or $\phi(w, z)<G(w, z)$, at such a point. Therefore, $G(w, z)$ is bounded outside $\Omega$. (Indeed, we have proved that $G(w, z)$, for $w$ outside $\Omega$, attains its maximum on the boundary of $\Omega$.)

We shall conclude with several remarks concerning the generality of the method. First, the considerations apply equally well to an arbitrary elliptic partial differential equation satisfying a maximum principle. All that is required is the construction of a parametrix, and this is done in a standard manner using a number of terms of a Neumann expansion analogous to (23). Also, the construction of a particular function analogous to $v_{3}$ can be performed under reasonable assumptions concerning the behavior of the coefficients of the differential equation on the boundary $C$. The fact that the boundary values of $G$ are zero, as in $\S 3$, can be obtained by using the infinite Neumann series for $S_{0}^{*}$. Of course, if the equation is not self-adjoint, the adjoint equation will enter into the considerations.

Second, the considerations apply to Riemannian manifolds in place of domains in Euclidian space. Integrals of the first, second, and third kinds, with or without periods, can be constructed.

\section{REFERENCES}

1. S. Banach, Théorie des opérations linéaires, Warsaw, 1932.

2. R. Caccioppoli, Sui teoremi d'esistenza di Riemann, Annali della Scuola Normale Superiore di Pisa. Scienze Fisiche e Matematiche, Ser. II, vol. 7 (1938) pp. 177-187.

3. P. R. Garabedian, The classes $L_{p}$ and conformal mapping, Trans. Amer. Math. Soc. vol. 69 (1950) pp. 392-415.

4. P. R. Garabedian and M. Schiffer, On existence theorems of potential theory and conformal mapping, Ann. of Math. vol. 52 (1950) pp. 164-187. 526-531.

5. P. D. Lax, On the existence of Green's function, Proc. Amer. Math. Soc. vol. 3 (1952) pp.

6. C. Miranda, Sul principio di Dirichlet per le funzioni armoniche, Atti della Accademia Nazionale dei Lincei. Rendiconti, Classe di Scienze Fisiche, Matematiche e Naturali vol. 3 (1947) pp. 55-59.

STANFORD UNIVERSITY, Stanford Calif. 